\title{
Role of fetal ultrasound in prenatally diagnosed de novo balanced translocations
}

\author{
Eui Sun Seong, ${ }^{1}$ Hye Jin Youn', Min Kyung Park', Hye Yeon Boo', Bom Yi Lee², Hyun Mee Ryu ${ }^{1,2}$, and You Jung Han ${ }^{1, *}$ \\ 'Department of Obstetrics and Gynecology, Cheil General Hospital and Women's Healthcare Center, Dankook University College of Medicine, \\ Seoul, Korea \\ ${ }^{2}$ Laboratory of Medical Genetics, Cheil General Hospital and Women's Healthcare Center, Seoul, Korea
}

Purpose: This study aimed to investigate fetal ultrasonographic findings in cases of prenatally diagnosed de novo balanced translocations and the role of fetal ultrasound in prenatal genetic counseling.

Materials and Methods: We collected cases with de novo balanced translocations that were confirmed in chorionic villus sampling, amniocentesis, and cordocentesis between 1995 and 2016. A detailed, high-resolution ultrasonography was performed for prediction of prognosis. Chromosomes from the parents of affected fetuses were also analyzed to determine whether the balanced translocations were de novo or inherited.

Results: Among 32,070 cases with prenatal cytogenetic analysis, 27 cases (1/1,188 incidence) with de novo balanced translocations were identified. Fourteen cases (51.9\%) showed abnormal findings, and the frequency of major structural anomalies was $11.1 \%$. Excluding the major structural anomalies, all mothers who continued pregnancies delivered healthy babies.

Conclusion: Results of a detailed, high-resolution ultrasound examination are very important in genetic counseling for prenatally diagnosed de novo balanced translocations.

Key words: Genetic translocation, Prenatal ultrasonography, Prenatal diagnosis, Genetic counseling, Cytogenetic analysis.

\section{Introduction}

Balanced translocations are rare and occur either de novo or through parental inheritance. Inherited balanced translocations have been reported to be less frequently associated with major congenital anomalies than de novo. In a previous study, the estimated risk of a major congenital anomaly was 1.96\% among those with inherited balanced chromosome rearrangements, which was similar to the estimated risk in the general population [1]. In contrast, the frequency of congenital abnormalities in de novo balanced chromosome rearrangements was reported as $6.1 \%$ to $6.7 \%$ and higher than inherited type $[1,2]$. Although there was related study published in Korea, there were only 5 cases of de novo balanced translocations [3]. These reference values were reported in the 1990s and early 2000s and are not representative of current developments in prenatal ultrasound diagnostic technology. Abnormal ultrasound findings are helpful in prediction of pregnancy outcomes. Especially, when chromosomal abnormalities are diagnosed prenatally, the results of ultrasound are used to predict postnatal prognosis in genetic counseling.

This study aimed to investigate fetal ultrasonographic find-

\footnotetext{
Received: May 24 2018, Revised: June 12 2018, Accepted: June 12 2018, Published: 30 June 2018

*Corresponding author: You Jung Han, M.D., Ph.D. (D) http://orcid.org/0000-0003-4087-9198

Department of Obstetrics and Gynecology, Cheil General Hospital and Women's Healthcare Center, Dankook University College of Medicine, 23 Toegyero 46-gil, Jung-gu, Seoul 04619, Korea.

Tel: +82-2-2000-7127, Fax: +82-2-2278-4574, E-mail: hanyj1978@hanmail.net

Conflict of interest: The authors declare that they do not have any conflicts of interest.

(c) This is an open-access article distributed under the terms of the Creative Commons Attribution Non-Commercial License (http://creativecommons.org/licenses/by-nc/4.0/) which permits unrestricted non-commercial use, distribution, and reproduction in any medium, provided the original work is properly cited.

(c) Copyright 2018 by the Korean Society of Medical Genetics and Genomics 
ings and pregnancy outcomes in cases of prenatally diagnosed de novo balanced translocations and to provide recent results that can be useful in genetic counseling.

\section{Materials and Methods}

\section{Study subjects}

We retrospectively reviewed the medical records of 32,070 pregnant women who underwent prenatal cytogenetic analysis in our institute between 1995 and 2016. The indications for prenatal cytogenetic analysis included an advanced maternal age, high risk for maternal serum screening test, abnormal ultrasound findings, parental chromosomal abnormalities, and other reasons. All fetuses with de novo balanced translocation were subjected to nuchal translucency ultrasound or a detailed, high-resolution ultrasound examination. Nuchal translucency ultrasound examined fetal anatomy and nuchal translucency thickness between 11 weeks and 13 weeks 6 days of gestation. A detailed, high-resolution ultrasound examination was performed between 18 and 24 weeks of gestation and assessed fetal anatomy systematically. Pregnant women who continued to receive antenatal screening in our institute performed ultrasound examination again in the third trimester of pregnancy. The babies were regarded as healthy if they showed normal appearances. For this study, appropriate institutional review board approval was obtained from the Ethics Committee at Cheil General Hospital (\#CGH-IRB-2017-50).

\section{Prenatal cytogenetic analysis}

Amniocentesis, chorionic villi sampling, and cordocentesis were performed to obtain specimens for the cytogenetic analysis. Cytogenetic analysis for fetal karyotyping were performed using the conventional GTG or GTL-banding analysis method. The chromosomes of parents of the affected fetuses were also analyzed to determine whether the balanced translocations either occurred de novo or were inherited. Peripheral blood of parents was used for cytogenetic analysis and the analysis was performed using same method as fetuses.

\section{Results}

Among the 32,070 available cases subjected to prenatal cy-

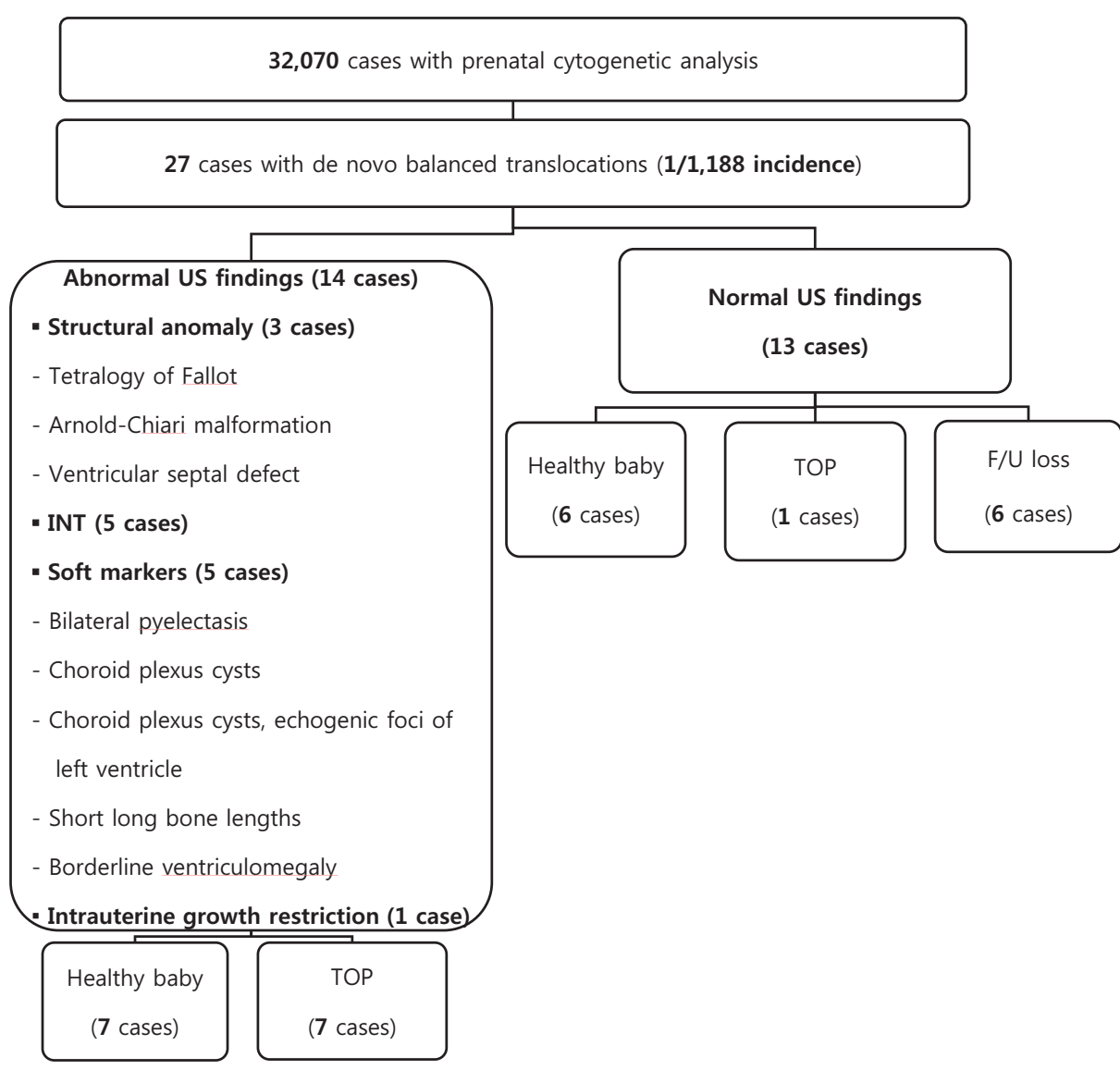

Fig. 1. Flowchart of the study. The incidence of prenatally diagnosed de novo balanced translocations was 1/1,188 at our institute. Abnormal ultrasound (US) findings were identified in 14 cases $(51.9 \%)$. The risk of major structural anomaly in prenatally diagnosed de novo balanced translocations was $11.1 \%$. INT, increased nuchal translucency; TOP, termination of pregnancy; F/U, follow up. 
togenetic analysis, 27 cases with de novo balanced translocations were identified. The incidence of prenatally diagnosed de novo balanced translocations was 1/1,188 at our institute. The frequencies of de novo balanced reciprocal translocation and Robertsonian translocation were 1/1,603 and 1/4,581 respectively. The indications for cytogenetic analysis were advanced maternal age in 14 cases, high risk for maternal serum screening test in 7 cases, and abnormal ultrasound findings in 6 cases. Amniocentesis in 25 cases, chorionic villi sampling in 1 case, and cordocentesis in 1 case were performed. Following confirmation of the results of cytogenetic analyses, detailed, high-resolution ultrasound examinations were performed in 21 cases.

Fig. 1 summarizes the results of 27 cases with de novo balanced translocations. Abnormal ultrasound findings were identified in 14 cases (51.9\%). The findings from these cases are summarized in Table 1. The abnormal ultrasound findings included 5 cases with increased nuchal translucency, 3 cases with major structural anomaly, 5 cases with soft markers, and 1 case with intrauterine growth restriction. The major structural anomalies included 2 cases with congenital heart diseases and 1 case with Arnorld-Chiari malformations. The risk of major structural anomaly in prenatally diagnosed de novo balanced translocations was $11.1 \%$ and all 3 cases such pregnancies were terminated. In 7 cases with ongoing pregnancy, all babies were delivered at term and phenotypically normal.

Thirteen cases (48.1\%) showed normal ultrasound findings and pregnancy outcomes could be confirmed in 7 cases. Pregnancy was terminated in one case because of parental request; in the remaining 6 cases of ongoing pregnancy, all babies were delivered at term and were phenotypically normal (Table 2).

\section{Discussion}

Several reports have published the incidence of prenatally diagnosed de novo balanced translocations [1,2]. In 1991, the frequencies of approximately $1 / 2,000$ and 1/9,000 for de novo balanced reciprocal translocations and Robertsonian translocations, respectively [2]. According to a study published in 2006, the incidence of de novo balanced reciprocal translocations was $1 / 1,246$ and de novo Robertsonian translocations was 1/6,234 [1]. In our study, the incidence was higher; that may be due to not racial differences, but also advances in diagnostic techniques such as development of resolution of chromosome analysis and introduction of various additional confirmatory tests.

Balanced translocations are expected to have little effect on the phenotype due to the absence of genetic material changes, whether balanced reciprocal or Robertsonian translocations. In our study, we estimated major structural anomaly rates of

Table 1. Summary of 14 cases with abnormal ultrasound findings in prenatally diagnosed de novo balanced translocations

\begin{tabular}{|c|c|c|c|}
\hline Karyotype & Ultrasound findings & Indication of karyotyping & Pregnancy outcomes \\
\hline \multicolumn{4}{|l|}{ Reciprocal translocation } \\
\hline $46, X X, t(8 ; 20)(q 24.2 ; q 12)$ & $\begin{array}{l}\text { Arnold-Chiari malformations } \\
\text { Thickened myocardium }\end{array}$ & AMA & TOP \\
\hline $46, X X, t(2 ; 4)(p 23 ; q 28)$ & Ventricular septal defect, MR, AR & Ab-US & TOP \\
\hline $46, X X, t(3 q ; 7 q ; 12 q)$ & INT & Ab-US & TOP \\
\hline 46,XY,t(3;16)(p13;q25) & INT & Ab-US & TOP \\
\hline 46,XY,t(2;20)(q12;p12) & INT & T21 HR on MSS & TOP \\
\hline 46,XX,t(8;10)(q22.1;p13) & INT & $A b-U S$ & $3,105 \mathrm{~g}$ healthy baby \\
\hline 46,X,t(X;10)(q26;q11.2) & Pyelectasis & T18 HR on MSS & TOP \\
\hline $46, X Y, t(7 q ; 14 q)$ & $\mathrm{CPC}$ & AMA & $3,440 \mathrm{~g}$ healthy baby \\
\hline $\begin{array}{l}46, X, t(X ; 14)(p 11.4 ; q 13)[15] \\
/ 46, X X[60]\end{array}$ & CPC, LV echogenic foci & Ab-US & 3,255 g healthy baby \\
\hline 46,XX,t(6;18)(q25.1;q23) & Short long bone lengths & AMA & $3,260 \mathrm{~g}$ healthy baby \\
\hline 46,XY,t(9;13)(p21;q32) & Borderline ventriculomegaly & AMA & $3,440 \mathrm{~g}$ healthy baby \\
\hline 46,XX,t(1;15;5)(p32.3;q21.1;q31.3) & IUGR & AMA & 2,290 g healthy baby \\
\hline \multicolumn{4}{|l|}{ Robertsonian translocation } \\
\hline 45,XY,der(13;14)(q10;q10) & INT & Ab-US & $3,030 \mathrm{~g}$ healthy baby \\
\hline $45, X X, \operatorname{der}(13 ; 14)(q 10 ; q 10)$ & TOF & T21 HR at MSS+ & TOP \\
\hline
\end{tabular}

AMA, advanced maternal age; TOP, termination of pregnancy; MR, mitral regurgitation; AR, aortic regurgitation; Ab-US, abnormal ultrasound; INT, increased nuchal translucency; T, trisomy; HR on MSS, high risk on maternal serum screening; CPC, choroid plexus cyst; LV, left ventricle; IUGR, intrauterine growth restriction; TOF, tetralogy of Fallot. 
Table 2. Summary of 13 fetuses with normal ultrasound findings in prenatally diagnosed de novo balanced translocations

\begin{tabular}{|c|c|c|}
\hline Karyotype & $\begin{array}{l}\text { Indication of } \\
\text { karyotyping }\end{array}$ & Pregnancy outcomes \\
\hline \multicolumn{3}{|l|}{ Reciprocal translocation } \\
\hline 46,XX,t(2;21)(q11.1;q22.3) & AMA & $3,705 \mathrm{~g}$ healthy baby \\
\hline $46, X Y, t(10 ; 19)$ & T21 HR on MSS & 3,855 g healthy baby \\
\hline 46,X,t(Y;11)(q1?1.2;q2?3) & T21 HR on MSS & $3,655 \mathrm{~g}$ healthy baby \\
\hline 46,XX,t(2;6)(p24;q23.3) & T21 HR on MSS & $3,545 \mathrm{~g}$ healthy baby \\
\hline $46 X X, t(17 q ; 22 p)$ & AMA & TOP \\
\hline 46,XY,t(11;22)(q23;q11.2) & AMA & Lost to follow-up \\
\hline 46,XX,t(7;15)(q36.1;q15) & AMA & Lost to follow-up \\
\hline \multicolumn{3}{|l|}{ Robertsonian translocation } \\
\hline 45,XY,der(13;13)(q10;q10) & AMA & 2,905 g healthy baby \\
\hline 45,XY,der(13;14)(q10;q10) & AMA & $3,410 \mathrm{~g}$ healthy baby \\
\hline 46,XX,t(14;15)(q24.3;q26.2) & AMA & Lost to follow-up \\
\hline $46, X Y, t(13 ; 21)$ & AMA & Lost to follow-up \\
\hline $45, X X, \operatorname{der}(14 ; 15)(q 10 ; q 10)$ & AMA & Lost to follow-up \\
\hline 45,XX,der(13;15)(q10;q10) & T21 HR on MSS & Lost to follow-up \\
\hline
\end{tabular}

AMA, advanced maternal age; T, trisomy; HR on MSS, high risk on maternal serum screening; TOP, termination of pregnancy.

$10.5 \%$ and $12.5 \%$ for de novo balanced reciprocal and Robertsonian translocations, respectively. However, these frequencies may have been overestimated. Our analysis included 6 cases wherein a cytogenetic diagnosis was performed because of abnormal ultrasound findings. Therefore, we recalculated the risk, as these cases would have introduced ascertainment bias. After excluding the 6 cases, we determined major structural anomaly rates of 7.1\% for de novo balanced reciprocal translocations, 14.2\% for de novo Robertsonian translocations, and 9.5\% for all de novo balanced translocations. A previous study reported frequencies of serious congenital anomaly of $6.1 \%$ for de novo balanced reciprocal translocations and 3.7\% for Robertsonian translocations; these serious congenital anomalies included neurodevelopmental disorders, as well as major structural anomalies [2]. Peng et al. [1] reported a major structural anomaly rate of 8.3\% among cases of prenatally detected de novo balanced translocations. These figures were higher than the usual estimated frequency of 2\% to $3 \%$ for congenital anomalies at birth. This discrepancy may be attributed to the mechanisms by which gene disruption at chromosomal breakpoints cause abnormal gene function [4]. Therefore, the ultrasound examination should be carefully performed considering a high incidence of major congenital anomaly if a de novo balanced translocation is confirmed prenatally.

Prenatal genetic counseling for de novo balanced translocations should include both neurodevelopment disorders and structural anomalies. Short-term follow-up studies reported frequencies of $6.1 \%$ to $12.5 \%$ for congenital abnormalities among cases of de novo balanced chromosome rearrangements [1,3,58]. Sinnerbrink et al. [9] evaluated the long-term follow-up data of children aged 3 to 11 years who have been diagnosed with de novo balanced chromosomal rearrangements. The results of this study were as follows: In children with a prenatally detected de novo balanced chromosomal rearrangement, there was no significant differences of intelligence, educational ability, mental health, and child development compared with general population. In our study, we did not investigate a long-term follow-up of the affected babies. However, after excluding cases involving major structural anomalies, all mothers with ongoing pregnancies gave birth to healthy babies.

The development of next-generation sequencing (NGS) technologies or chromosomal microarray has allowed cases previously classified as de novo balanced translocations to be identified as unbalanced translocations. Among individuals with postnatally diagnosed de novo balanced chromosomal rearrangements and abnormal phenotypes, 40\% to 100\% are found to have a genomic imbalance detectable by a comparative genomic hybridization array or single-nucleotide polymorphism array [10-12]. In the future, re-analyses of de novo balanced translocations and ultrasound results by applying NGS technology is expected to yield more interesting results.

In summary, we concluded that the frequency of major congenital anomalies prenatally diagnosed de novo balanced translocations was as high as 10\%, but the prognosis of the affected fetuses without major anomalies did not significantly differ from those of the general population. Therefore, our results suggest that the results of a detailed, high-resolution ultrasound examination are a very important factor in genetic counseling for prenatally diagnosed de novo balanced translocations.

\section{Acknowledgements}

This research was supported by a grant of the Korean Health Technology R\&D Project through the Korean Health Industry Development Institute (KHIDI), funded by the Ministry of Health \& Welfare, Republic of Korea (grant number: HC15C1336).

\section{References}

1. Peng HH, Chao AS, Wang TH, Chang YL, Chang SD. Prenatally diagnosed balanced chromosome rearrangements: eight years' experience. J Reprod Med 2006;51:699-703. 
2. Warburton D. De novo balanced chromosome rearrangements and extra marker chromosomes identified at prenatal diagnosis: clinical significance and distribution of breakpoints. Am J Hum Genet 1991;49:995-1013.

3. Park S, Lee BY, Kim YM, Kim JM, Lee MH, Kim JW, et al. De novo chromosomal aberrations in the fetus; genetic counseling and clinical outcome. J Korean Med Sci 2003;18:397-401.

4. Talkowski ME, Ordulu Z, Pillalamarri V, Benson CB, Blumenthal I, Connolly $S$, et al. Clinical diagnosis by whole-genome sequencing of a prenatal sample. N Engl J Med 2012;367:2226-32.

5. Wassman ER, Cheyovich DL, Nakahara Y. "Possibly" de novo translocations: prenatal risk counseling. Am J Obstet Gynecol 1989;161:698702.

6. MacGregor DJ, Imrie S, Tolmie JL. Outcome of de novo balanced translocations ascertained prenatally. J Med Genet 1989;26:590-1.

7. Chen CP, Wu PC, Lin CJ, Su YN, Chern SR, Tsai FJ, et al. Balanced reciprocal translocations detected at amniocentesis. Taiwan J Obstet Gynecol 2010;49:455-67.

8. Tierney I, Axworthy D, Smith L, Ratcliffe SG. Balanced rearrangements of the autosomes: results of a longitudinal study of a newborn survey population. J Med Genet 1984;21:45-51.

9. Sinnerbrink IB, Sherwen A, Meiser B, Halliday J, Amor DJ, Waters E, et al. Long-term health and development of children diagnosed prenatally with a de novo apparently balanced chromosomal rearrangement. Prenat Diagn 2013;33:831-8.

10. Schluth-Bolard C, Delobel B, Sanlaville D, Boute O, Cuisset JM, Sukno $S$, et al. Cryptic genomic imbalances in de novo and inherited apparently balanced chromosomal rearrangements: array CGH study of 47 unrelated cases. Eur J Med Genet 2009;52:291-6.

11. Gijsbers AC, Bosch CA, Dauwerse JG, Giromus O, Hansson K, HilhorstHofstee $Y$, et al. Additional cryptic CNVs in mentally retarded patients with apparently balanced karyotypes. Eur J Med Genet 2010;53:22733.

12. De Gregori M, Ciccone $R$, Magini P, Pramparo T, Gimelli S, Messa J, et al. Cryptic deletions are a common finding in "balanced" reciprocal and complex chromosome rearrangements: a study of 59 patients. J Med Genet 2007;44:750-62. 